\title{
A Clinician's Guide to the Recognition and Management of Dupilumab-Associated Conjunctivitis
}

\author{
Gaurav Agnihotri ${ }^{1} \cdot$ Katherine Shi ${ }^{2} \cdot$ Peter A. Lio ${ }^{2,3}$
}

Published online: 14 November 2019

(c) The Author(s) 2019

\begin{abstract}
Dupilumab, a monoclonal antibody that inhibits both interleukin (IL)-4 and IL-13 signaling, is an effective treatment option in moderate-to-severe atopic dermatitis (AD). Patients with $\mathrm{AD}$ are already at increased risk of developing conjunctivitis, and clinical trials and case reports have shown a greater incidence of conjunctivitis in individuals with AD treated with dupilumab. As this is one of the more commonly reported side effects of this biologic agent, it is important that clinicians are aware of this association and advise patients receiving dupilumab to report signs of conjunctivitis. This review summarizes the risk factors, clinical features, and management options for patients with $\mathrm{AD}$ presenting with conjunctivitis after receiving dupilumab therapy.
\end{abstract}

\section{Key Points}

Patients receiving dupilumab for atopic dermatitis may be at increased risk of developing conjunctivitis.

This article provides clinical guidance on the recognition and management of dupilumab-associated conjunctivitis.

The approach to management involves distinguishing between mild and moderate-to-severe conjunctivitis to tailor therapy appropriately, and co-management with ophthalmology is often required.

\section{Introduction}

Atopic dermatitis (AD) is a chronic inflammatory skin condition that can be challenging to treat [1]. A variety of therapies for $\mathrm{AD}$ are available, ranging from topical agents such as corticosteroids, calcineurin inhibitors, and

Peter A. Lio

peterlio@gmail.com

1 University of Illinois at Chicago College of Medicine, Chicago, IL, USA

2 Department of Dermatology, Feinberg School of Medicine, Northwestern University, Chicago, IL, USA

3 Medical Dermatology Associates of Chicago, 363 W Erie Street, Suite 350, Chicago, IL 60654, USA phosphodiesterase inhibitors to systemic immunosuppressants such as cyclosporine, methotrexate, azathioprine, and mycophenolate mofetil. Prednisone is the only systemic immunosuppressant approved for the treatment of $\mathrm{AD}$ in the USA, whereas cyclosporine is approved in other countries [2, 3]. In 2017, the US FDA approved dupilumab, the first human monoclonal antibody for the treatment of AD, which works by inhibiting the alpha subunit of interleukin (IL)-4, subsequently blocking downstream signaling of IL-4 and IL-13 [4]. In the USA, dupilumab is administered subcutaneously at a dosage of 200-300 mg every 2 weeks for patients aged $\geq 12$ years with moderate-to-severe AD that is uncontrolled with topical therapies or for when those therapies are contraindicated [4].

Conjunctivitis is one of the more common adverse effects of dupilumab. Clinicians who use dupilumab to treat patients with AD should be aware of the signs and symptoms of and the management options for conjunctivitis that may subsequently develop. However, no standard guidelines exist on how to diagnose and treat conjunctivitis in patients receiving dupilumab. This article presents an overview of dupilumabassociated conjunctivitis (DAC) epidemiology, risk factors, and theorized mechanisms for its development. This is followed by a brief review for dermatologists and other clinicians of the common clinical presentations and management options observed through case studies and clinical trials. As this is a rapidly changing area, we build upon knowledge summarized in prior reviews. Since the last review by Aszodi et al. [35], 11 case series and reports regarding conjunctivitis and ocular surface disease related to dupilumab treatment and AD have been published and are included in 
this paper. Only nine case series and reports characterize ocular findings in DAC; these are described in Table 1.

\section{Methods}

A search of the PubMed database for case reports and clinical trials using the keywords ("dupilumab" and "atopic dermatitis") or ("dupilumab" and "conjunctivitis") yielded 312 papers. Articles published before 31 July 2019 relating to dupilumab and ocular surface diseases were reviewed. After removing duplicate articles, we screened 233 papers by title and 60 papers by abstract. Articles were excluded if the paper was not written in the English language. Articles were included if the primary assessment was dupilumab treatment of $\mathrm{AD}$ in adults or ocular surface disease in adult patients with AD receiving dupilumab. Papers describing the pathophysiology and epidemiology of DAC were also included. We then conducted a full-text screen of 43 articles. A total of 29 studies were included in this review, including six randomized controlled trials and 11 case reports. See Fig. 1 for study selection details.

\section{Epidemiology and Risk Factors}

Akinlade et al. [8] analyzed six randomized, double-blinded, placebo-controlled clinical trials in patients with $\mathrm{AD}$ treated with dupilumab, which yielded a cohort of 2629 patients, providing the most in-depth examination into the incidence and risk factors for developing DAC as of the end date of the literature search performed for this review [5-10]. Overall, patients treated with dupilumab had a higher incidence of conjunctivitis (8.6-22.1\%) than did those receiving placebo (2.1-11.1\%) for all except one trial (SOLO-CONTINUE) [8]. The conjunctivitis in these patients was mostly mild-tomoderate in severity. These cases were assessed by the clinical trial investigators, who were dermatologists or allergists, and were not evaluated by ophthalmologists. Moreover, the reports of conjunctivitis did not differentiate between allergic, infectious, or chemical types. In total, $<0.5 \%$ of patients with $\mathrm{AD}$ treated with dupilumab and $<0.3 \%$ of patients treated with placebo had severe conjunctivitis [8].

Certain risk factors are also associated with the development of conjunctivitis in patients with $\mathrm{AD}$, independent of dupilumab use. In the same analysis by Akinlade et al. [8], patients with more severe AD at baseline [Investigator's Global Assessment (IGA) score =4] were more likely to report a higher incidence of conjunctivitis regardless of whether they received dupilumab or placebo. This was also seen in a case series by Treister et al. [11], where 9 of $12(75 \%)$ patients with DAC had more severe disease at baseline (IGA score of 4). Increased rates of conjunctivitis have previously been associated with $\mathrm{AD}$ severity $[12,13]$. Moreover, according to Akinlade et al. [8], patients with a self-reported history of conjunctivitis or with an increased baseline level of serum biomarkers, such as thymus and activation-regulated chemokine, immunoglobulin $\mathrm{E}$, or eosinophils, had a higher incidence of conjunctivitis regardless of whether they received dupilumab or placebo.

Remarkably, this adverse event appears to be limited to those with AD: current data from randomized, double-blinded, placebo-controlled clinical trials suggest no significant increase in the incidence of conjunctivitis in dupilumab-treated asthma [14-16], chronic rhinosinusitis with nasal polyps [17], or eosinophilic esophagitis [18] compared with placebo. The overall incidence of conjunctivitis in these three patient populations is lower than in those with AD: $0-1.7 \%$ in dupilumab-treated groups and $0-3.3 \%$ in placebo-treated groups [8, 14-18].

\section{Pathogenesis}

The pathogenesis of conjunctivitis in dupilumab-treated patients is not well-understood, but several theories have been postulated: reduction in ocular cytokines provides a favorable environment for Demodex mites to grow, causing IL-17-mediated inflammation [19]; eosinophilia after dupilumab administration [12]; increased downstream activity of the OX40 ligand in the eye [20]; and systemic IL-13 inhibition indirectly leading to a reduction in conjunctival goblet cells and mucin production [21]. Further studies are required to explain the disproportionally higher incidence of conjunctivitis in patients with AD treated with dupilumab compared with those with asthma, chronic rhinosinusitis, or eosinophilic esophagitis.

\section{Clinical Presentation}

Several case reports and clinical trials have described features characteristic of the vast majority of DAC (Table 1) [11, 22-27]. The most prominent finding-bilateral conjunctival hyperemia - was present in every patient, with certain cases noting limbal hyperemia in particular [22, 25, 26]. Ocular symptoms included pruritus, tearing, irritation, foreign body sensation, and decreased visual acuity in some but not all patients [11, 22, 23, 28]. These symptoms are commonly present in those with conjunctivitis [28]. Other clinical patterns included cicatrizing conjunctivitis [26, 27] and follicular conjunctivitis [23, 25].

The clinical signs of conjunctivitis can often be nonspecific, and it may be difficult to differentiate between the different types of conjunctivitis. Dry eye can also present 


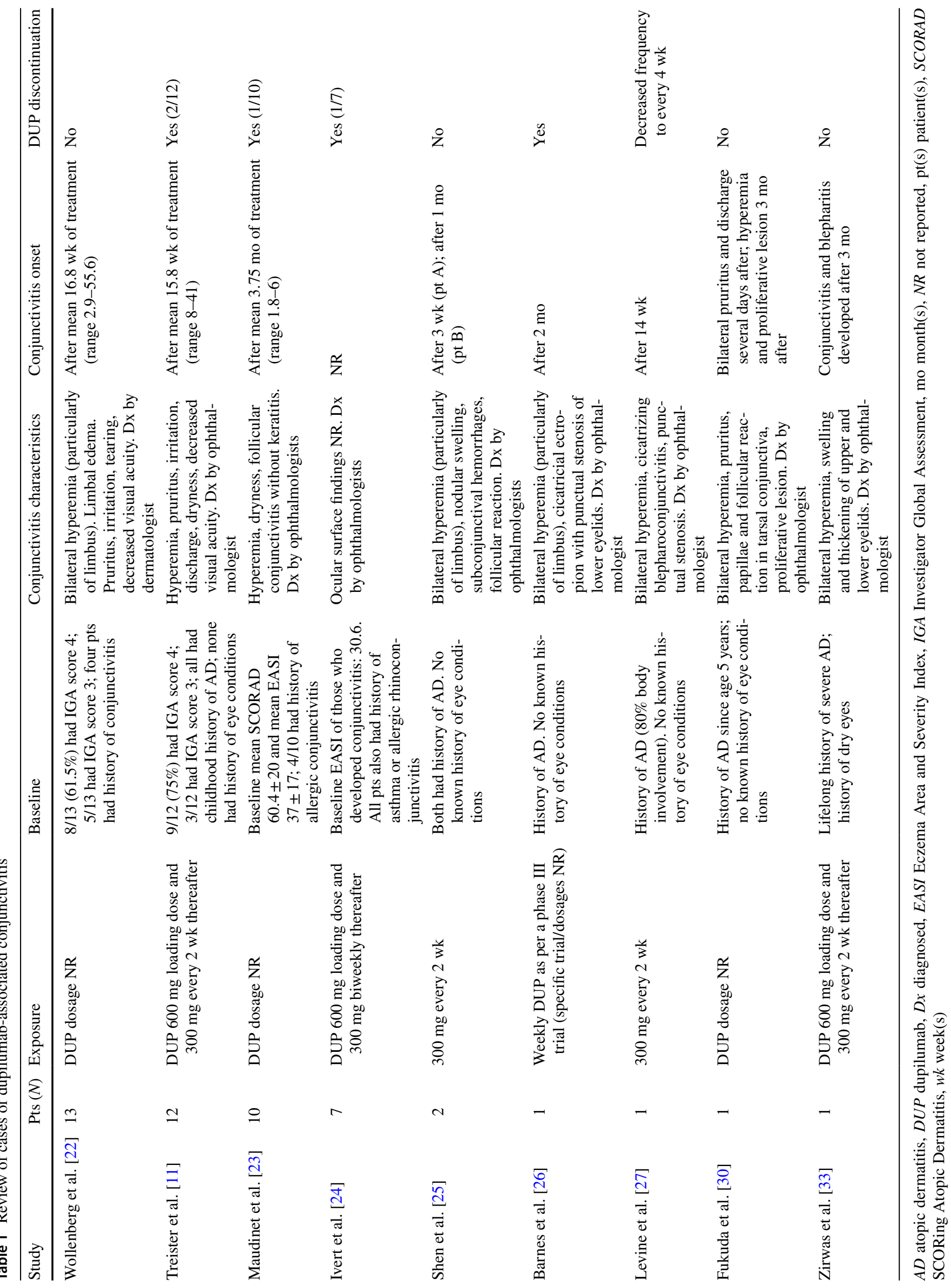



ing the study selection process. Identified papers describe dupilumab treatment of atopic dermatitis and dupilumab-associated conjunctivitis
Fig. 1 PRISMA diagram detail-
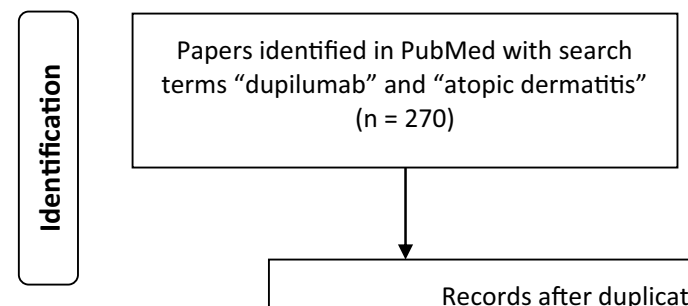

Papers identified in PubMed with search terms "dupilumab" and "conjunctivitis" $(n=42)$
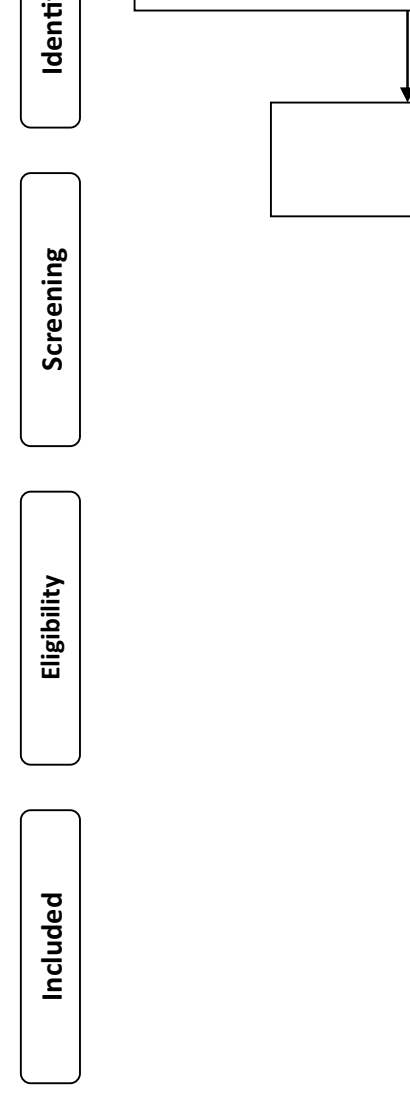
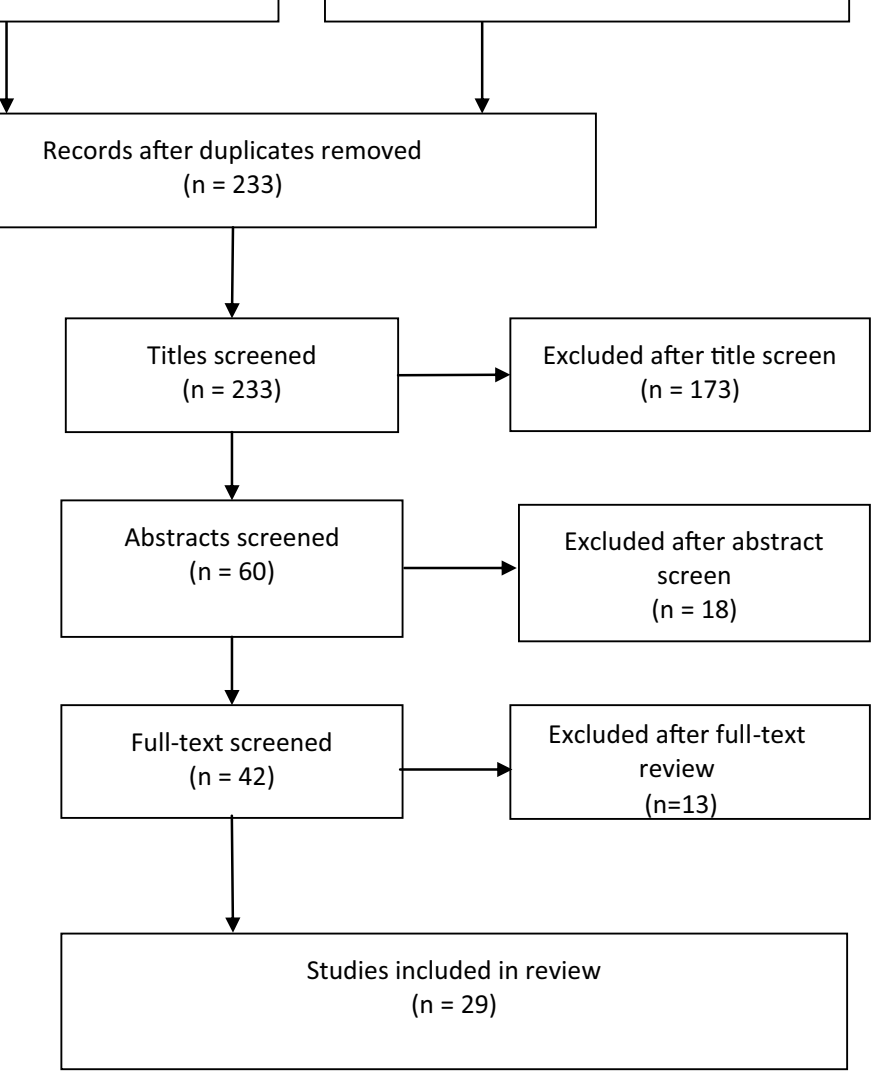

similarly to conjunctivitis $[28,29]$. Other clinical diagnoses for the patient presenting with conjunctival hyperemia that must be considered because of a risk of loss of vision include episcleritis, scleritis, keratitis, iritis, uveitis, and acute glaucoma [28].

Onset of conjunctivitis from dupilumab administration varied from weeks to months (Table 1) [11, 22-27]. In the trials analyzed by Akinlade et al. [8], the mean time from dupilumab initiation to conjunctivitis development was 2 weeks in the monotherapy pool (those who received only dupilumab, $n=1047$ ), $6-8$ weeks in the CHRONOS trial (those who received dupilumab and topical corticosteroids, $n=425$ ), and 4-8 weeks in the CAFÉ trial (those who received dupilumab and topical corticosteroids, $n=217$ ) [8]. The rate of new conjunctivitis appearance was constant throughout the 16-week studies (monotherapy pool and CAFÉ). In the CHRONOS trial, the number of new cases stabilized by weeks 20-24 [8].

Discontinuation of dupilumab was not commonly reported but was described in a few cases of severe conjunctivitis $[11,23,24,26]$. In the case series by Triester et al. [11], two patients who discontinued dupilumab experienced an improvement in conjunctivitis severity, examined at 8 and 10 weeks, but not a complete resolution, as bilateral palpebral hyperemia

persisted. Additionally, in the case report by Maudinet et al. [23], the patient who discontinued dupilumab experienced an improvement in conjunctivitis 8 weeks later but not a complete resolution. While the mechanism of dupilumab elimination (dosed at $300 \mathrm{mg}$ every 2 weeks) is not well-characterized, it is suggested the median time to nondetectable concentrations is 10 weeks [4].

\section{Management Recommendations}

Data around the mechanism and subsequent management of DAC are lacking. Despite this, a pattern of treatments described in small case series and case reports has been shown to be successful for patients and corroborated by the clinical experience of the International Eczema Council (IEC), which consists of 86 AD experts from 22 countries [22-25, 27, 28, 30-35].

The approach to the treatment of DAC starts with differentiating between mild and moderate-to-severe conjunctivitis [32, 33]. Mild conjunctivitis can typically be managed conservatively with warm compresses and artificial tears, sodium hyaluronate, trehalose/hyaluronate tear 
substitute, or antihistamine eyedrops, whereas moderate or severe conjunctivitis necessitates anti-inflammatory eyedrops or ointments containing corticosteroids, calcineurin inhibitors, or cyclosporine [22, 23, 27, 28, 30, 31, 35]. If mild cases of conjunctivitis do not resolve with conservative management, it would be appropriate to step up to a trial of therapies as outlined above for moderate-to-severe conjunctivitis.

No particular advantage of using one anti-inflammatory eyedrop over another is evident in terms of efficacy, although most clinicians prefer topical corticosteroids or calcineurin inhibitors initially. Of the corticosteroid eyedrops, fluorometholone $0.1-1 \%$ is more commonly used because of its poor penetration into the anterior chamber of the eye compared with other topical steroids, yielding a lower risk of cataract and glaucoma [22,35]. Other steroid eyedrops that have been used for DAC include hydrocortisone, dexamethasone, and loteprednol, although stronger steroids with greater penetration into the anterior chamber of the eye than fluorometholone should be used with caution [22, 23, 27, 32]. Topical steroids also carry a risk of increasing the latency of adenovirus and worsening corneal ulcers from herpetic, bacterial, or fungal infections, underscoring the importance of correctly diagnosing the type of conjunctivitis initially and co-management with an ophthalmologist [28]. The threshold for referral for ophthalmologic evaluation should be low when patients first develop signs and symptoms of conjunctivitis and when escalating therapy.

Tacrolimus $0.03-0.1 \%$ eyedrop or eye ointment is the only topical calcineurin inhibitor mentioned in the literature for treatment of DAC [22, 23, 27, 28, 30, 31, 35]. Patients reportedly respond very well to this treatment, and it has an additional benefit in that it can be used long term without a risk of developing cataracts or glaucoma. Similarly, cyclosporine eyedrops can also be used long term, as cyclosporine does not enter the anterior chamber of the eye, but it can cause a burning sensation upon application [23, 27, 32, 33]. Cyclosporine exerts an immunomodulatory effect on the ocular surface and increases conjunctival goblet cell density while decreasing corneal cell apoptosis by lowering the osmolarity of the tear film [23]. Increased tear film osmolarity stimulates inflammatory cytokine release, which increases the rate of cell apoptosis and decreases the number of goblet cells [23]. Topical tacrolimus or cyclosporine applied to the ocular surface can be used for maintenance after the acute conjunctivitis episode has resolved, especially if the patient cannot be tapered off the medicated eyedrops without recurrence of conjunctivitis [27].

Other regimens described in two case reports included "olopatadine $0.2 \%$, cyclosporine $0.5 \%$, and prednisolone acetate $1 \%$ eyedrops" [25] and "lifitegrast, cyclosporine $0.05 \%$, and loteprednol eyedrops" [33]. The former regimen yielded complete resolution of follicular conjunctivitis without recurrence in one patient and sufficient symptom control in another patient to allow for continued dupilumab injections [25]. These two patients were receiving long-term olopatadine $0.2 \%$ once daily, cyclosporine $0.05 \%$ twice daily, and prednisolone acetate $1 \%$ four times daily tapered over 4 days [25]. The patient without full conjunctivitis resolution required a prednisolone acetate $1 \%$ taper after every dupilumab injection [25]. In the latter regimen, the patient's conjunctivitis completely resolved with lifitegrast twice daily, cyclosporine $0.05 \%$ twice daily, and loteprednol twice daily (tapered to once daily after 6 weeks) [33]. Lifitegrast binds lymphocyte function-associated antigen-1 on white blood cells to block their interaction with intercellular adhesion molecule- 1 on vascular endothelial cells to prevent white blood cell extravasation from the vascular space into tissue [33]. Lifitegrast is currently FDA approved for chronic dry eye, but further studies are needed to confirm whether it can effectively be used in managing DAC [33].

In the vast majority of DAC cases, patients responded well and could continue to receive dupilumab injections. Only a handful of patients had to discontinue dupilumab because of severe or unresolving conjunctivitis, although several reports cited increasing the spacing between dupilumab injections to ameliorate this adverse effect [11, $23,24,26,27]$. A patient who developed cicatrizing blepharoconjunctivitis decreased the dose frequency of dupilumab from every 2 to every 4 weeks and applied dexamethasone $0.1 \%$ eyedrops four times daily, yielding improvement within 1 month [27].

After reviewing the literature for successful therapies for DAC, a suggested workflow of management is as follows and outlined in Fig. 2. At the first sign of new-onset conjunctivitis in patients with $\mathrm{AD}$ receiving dupilumab, a referral to ophthalmology for diagnosis, treatment recommendations, and monitoring should be considered. Hyperemia is the most important and prominent clinical sign of conjunctivitis; pruritus, tearing, irritation, and a foreign body sensation further support the clinical diagnosis [28]. Artificial tears or oral antihistamines can be started before referral, but dupilumab generally does not need to be discontinued before evaluation by ophthalmology. Prompt referral to ophthalmology is also indicated for any concerns about vision changes, eye pain, purulent discharge, corneal involvement, conjunctival scarring, recurrent episodes of conjunctivitis, history of herpes simplex eye infection, or clinical suspicion for another etiology [28]. Because of these considerations, the use of corticosteroid, tacrolimus, or cyclosporine eyedrops or ointment is probably best left to the discretion of and management by the ophthalmologist. If an ophthalmology consult cannot be obtained quickly, and treatment warrants corticosteroid eyedrops, consensus recommendation from the IEC is to initiate treatment before ophthalmic evaluation [31]. 


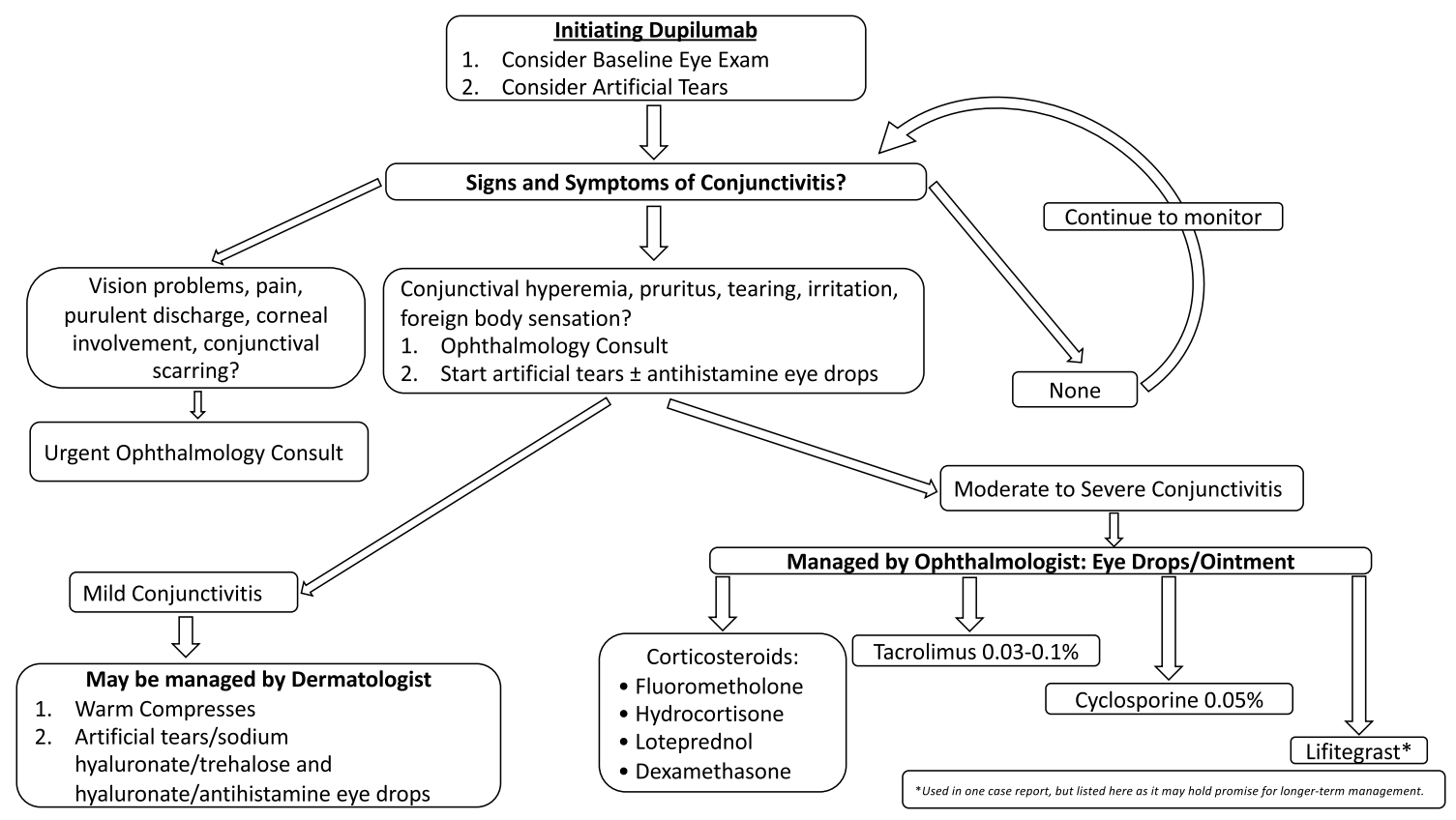

Fig. 2 Dupilumab-associated conjunctivitis management algorithm

In terms of preventive management, it is not unreasonable to suggest an eye exam by an ophthalmologist for patients with AD before initiating dupilumab given the high incidence of asymptomatic surface ocular disease at baseline and symptomatic surface ocular disease even without dupilumab treatment. Despite a small cohort of 25 patients, Maudinet et al. [23] showed that, before starting treatment with dupilumab, a baseline eye exam by an ophthalmologist identified 16 of $25(64 \%)$ patients with AD with an abnormal ocular surface. These patients were subsequently treated with warm compresses, artificial tears, and antihistamine drops, and-after 3 months of dupilumab-only 3 of 25 (12\%) developed conjunctivitis [23]. Rial et al. [34] also showed that prophylactic treatment with artificial tears every $12 \mathrm{~h}$ for 1 week before initiating dupilumab therapy resulted in just one of ten patients with $\mathrm{AD}$ developing superficial punctate keratitis after 12 weeks of dupilumab therapy. This patient also had a history of keratitis, with the last episode occurring 2 years before that reported in the study [34]. Since the follow-up duration was 12 weeks, it is possible that the incidence of conjunctivitis or keratitis could increase if the window of follow-up was extended. Data are currently insufficient to recommend an eye exam by an ophthalmologist for each patient initiating dupilumab treatment. However, this may change as more studies with larger sample sizes are published; ideally, a higher-risk subpopulation will be identified to undergo routine ophthalmologic exams before starting dupilumab. Conversely, ocular surface hydration with artificial tears is relatively benign and a reasonable prophylactic recommendation for patients with $\mathrm{AD}$ starting dupilumab therapy. This recommendation is also supported by some members of the IEC [31].

Lastly, it is important to note that previous history of conjunctivitis in patients with $\mathrm{AD}$ is not a contraindication to initiating dupilumab, a position with consensus agreement from members of the IEC [31].

\section{Conclusion}

Conjunctivitis is one of the more common side effects of dupilumab, and those with moderate-to severe AD are already predisposed to higher rates of conjunctivitis [11]. Whether dupilumab directly causes increased rates of conjunctivitis in patients with AD or whether higher rates of conjunctivitis are seen in patients with AD because they are already at a higher risk for developing conjunctivitis remains unknown [11,28]. Regardless, it is important for clinicians to be aware of both the symptoms of conjunctivitis and the management options, including when to refer to ophthalmology. Conjunctivitis should be considered when a patient with $\mathrm{AD}$ receiving dupilumab presents with bilateral hyperemic and pruritic eyes. Ophthalmology consult to confirm the diagnosis is recommended, and comanagement with ophthalmology is necessary if treatment requires steroid, tacrolimus, or cyclosporine eyedrops or eye ointment. As AD has a profound effect on quality of life, and those receiving dupilumab are part of the subset of patients with $\mathrm{AD}$ with disease that is difficult to control, discontinuing dupilumab altogether is not a desirable 
outcome. Fortunately, dupilumab discontinuation and even dose frequency spacing has rarely been needed to control DAC. With early and accurate diagnosis and proper treatment, patients with $\mathrm{AD}$ with DAC have a higher likelihood of being able to continue dupilumab and possibly achieve a successful reduction in symptom burden.

\section{Compliance with Ethical Standards}

Conflict of interest Gaurav Agnihotri and Katherine Shi have no conflicts of interest that are directly relevant to the content of this article. Peter A. Lio has served as an investigator, speaker, advisory board member, and consultant for Sanofi Genzyme/Regeneron.

Funding No sources of funding were used to conduct this study or prepare this manuscript.

Open Access This article is distributed under the terms of the Creative Commons Attribution-NonCommercial 4.0 International License (http://creativecommons.org/licenses/by-nc/4.0/), which permits any noncommercial use, distribution, and reproduction in any medium, provided you give appropriate credit to the original author(s) and the source, provide a link to the Creative Commons license, and indicate if changes were made.

\section{References}

1. Bieber T. Atopic dermatitis. N Engl J Med. 2008;358(14):1483-94.

2. Wollenberg A, Oranje A, Deleuran M, Simon D, Szalai Z, Kunz B, et al. ETFAD/ EADV eczema task force 2015 position paper on diagnosis and treatment of atopic dermatitis in adult and paediatric patients. J Eur Acad Dermatol Venereol. 2016;30(5):729-47.

3. Weidinger S, Novak N. Atopic dermatitis. Lancet. 2016;387(10023):1109-22.

4. Regeneron Pharmaceuticals. Dupixent (dupilumab) injection. https://www.accessdata.fda.gov/drugsatfda_docs/label /2017/761055lbl.pdf. Accessed 30 June 2019.

5. Simpson EL, Bieber T, Guttman-Yassky E, Beck LA, Blauvelt A, Cork MJ, et al. Two phase 3 trials of dupilumab versus placebo in atopic dermatitis. N Engl J Med. 2016;375(24):2335-48.

6. Blauvelt A, de Bruin-Weller M, Gooderham M, Cather JC, Weisman J, Pariser D, et al. Long term management of moderateto-severe atopic dermatitis with dupilumab and concomitant topical corticosteroids (LIBERTY AD CHRONOS): a 1-year, randomised, double-blinded, placebo-controlled, phase 3 trial. Lancet. 2017;389(10086):2287-303.

7. De Bruin-Weller M, Thaçi D, Smith CH, Reich K, Cork M, Radin A, et al. Dupilumab with concomitant topical corticosteroids in adult patients with atopic dermatitis who are not adequately controlled with or are intolerant to ciclosporin $\mathrm{A}$, or when this treatment is medically inadvisable: a placebo-controlled, randomized phase 3 clinical trial (LIBERTY AD CAFÉ). Br J Dermatol. 2018;178(5):1083-101.

8. Akinlade BO, Guttman-Yassky EM, de Bruin-Weller M, Simpson EL, Blauvelt A, Cork MJ, et al. Conjunctivitis in dupilumab clinical trials. Br J Dermatol. 2019. https://doi.org/10.1111/bjd.17869.

9. Thaci D, Simpson EL, Beck LA, Bieber T, Blauvelt A, Papp K, et al. Efficacy and safety of dupilumab in adults with moderateto-severe atopic dermatitis inadequately controlled by topical treatments: a randomised, placebo-controlled, dose-ranging phase 2b trial. Lancet. 2016;387(10013):40-52.

10. Worm M, Simpson EL, Thaci D et al. The effect of dose regimen adjustment on maintenance of clinical response and safety of dupilumab in patients with atopic dermatitis (LIBERTY AD SOLO-CONTINUE). Presented at the 37th annual congress of the European Academy of Allergy and Clinical Immunology (EAACI), Munich, 26-30 May 2018.

11. Treister AD, Kraff-Cooper C, Lio PA. Risk factors for dupilumabassociated conjunctivitis in patients with atopic dermatitis. JAMA Dermatol. 2018;154(10):1208-11.

12. Thyssen JP, Toft PB, Halling-Overgaard AS, Gislason GH, Skov L, Egeberg A. Incidence, prevalence, and risk of selected ocular disease in adults with atopic dermatitis. J Am Acad Dermatol. 2017;77(2):280-6.

13. Uchio E, Miyakawa K, Ikezawa Z, Ohno S. Systemic and local immunological features of atopic dermatitis patients with ocular complications. Br J Ophthalmol. 1998;82(1):82-7.

14. Wenzel S, Castro M, Corren J, Maspero J, Wang L, Zhang B, et al. Dupilumab efficacy and safety in adults with uncontrolled persistent asthma despite use of medium-to-high-dose inhaled corticosteroids plus a long-acting $\beta 2$ agonist: a randomised double-blind placebo-controlled pivotal phase $2 \mathrm{~b}$ dose-ranging trial. Lancet. 2016;388(10039):31-44.

15. Castro M, Corren J, Pavord I, Maspero J, Wenzel S, Rabe K, et al. Dupilumab efficacy and safety in moderate-to-severe uncontrolled asthma. N Engl J Med. 2018;378(26):2486-96.

16. Rabe KF, Nair P, Brusselle G, Maspero JF, Castro M, Zhu H, et al. Dupilumab in patients with corticosteroid-dependent severe asthma: efficacy and safety results from the randomized, doubleblind, placebo-controlled phase 3 LIBERTY ASTHMA VENTURE study. Am J Respir Crit Care Med. 2018;197:A7712.

17. Bachert C, Mannent L, Naclerio RM, Mullol J, Ferguson BJ, Gevaert P, et al. Effect of subcutaneous dupilumab on nasal polyp burden in patients with chronic sinusitis and nasal polyposis: a randomized clinical trial. JAMA. 2016;315(5):469-79.

18. Hirano I, Dellon ES, Hamilton JD, Collins MH, Peterson K, Chehade M. Dupilumab efficacy and safety in adult patients with active eosinophilic oesophagitis: a randomized double-blind placebo-controlled phase 2 trial. United Eur Gastroenterol J. 2017;5:1138-50.

19. Thyssen J. Could conjunctivitis in dupilumab treated atopic dermatitis patients be caused by colonization with Demodex and increased IL-17 levels? Br J Dermatol. 2018;178(5):1220.

20. Mennini M, Dahdah L, Fiocchi A, Simpson EL. Two phase 3 trials of dupilumab versus placebo in atopic dermatitis. N Engl J Med. 2017;376(11):1090-1.

21. Bakker DS, Ariens LFM, van Luijk C, van der Schaft J, Thijs JL, Schuttelaar MLA, et al. Goblet cell scarcity and conjunctival inflammation during treatment with dupilumab in patients with atopic dermatitis. Br J Dermatol. 2019;180(5):1248-9.

22. Wollenberg A, Ariens L, Thurau S, van Luijk C, Seegräber M, de Bruin-Weller M. Conjunctivitis occurring in atopic dermatitis patients treated with dupilumab-clinical characteristics and treatment. J Allergy Clin Immunol Pract. 2018;6(5):1778-80.

23. Maudinet A, Law-Koune S, Duretz C, Lasek A, Modiano P, Tran THC. Ocular surface diseases induced by dupilumab in severe atopic dermatitis. Ophthalmol Ther. 2019;8(3):485-90.

24. Ivert LU, Wahlgren CF, Ivert L, Lundqvist M, Bradley M. Eye complications during dupilumab treatment for severe atopic dermatitis. Acta Derm Venereol. 2019;99(4):375-8.

25. Shen E, Xie K, Jwo K, Smith J, Mosaed S. Dupilumab-induced follicular conjunctivitis. Ocular Immunol Inflamm. 2018. https:// doi.org/10.1080/09273948.2018.1533567. 
26. Barnes AC, Blandford AD, Perry JD. Cicatricial ectropion in a patient treated with dupilumab. Am J Ophthalmol Case Rep. 2017;7:120-2.

27. Levine RM, Tattersall IW, Gaudio PA, King BA. Cicatrizing blepharoconjunctivitis occurring during dupilumab treatment and a proposed algorithm for its management. JAMA Dermatol. 2018;154(12):1485-6.

28. Gooderham M, McDonald J, Papp K. Diagnosis and management of conjunctivitis for the dermatologist. J Cutan Med Surg. 2018;22(2):200-6.

29. Azari AA, Barney BP. Conjunctivitis: a systematic review of diagnosis and treatment. JAMA. 2013;310(16):1721-9.

30. Fukuda K, Ishida W, Kishimoto T, Fukushima A. Development of conjunctivitis with a conjunctival proliferative lesion in a patient treated with dupilumab for atopic dermatitis. Allergol Int. 2019;68(3):383-4.

31. Thyssen JP, de Bruin-Weller MS, Paller AS, Leshem YA, Vestergaard C, Deleuran M, et al. Conjunctivitis in atopic dermatitis patients with and without dupilumab therapy-international eczema council survey and opinion. J Eur Acad Dermatol Venereol. 2019;33(7):1224-31.

32. Beck KM, Seitzman GD, Yang EJ, Sanchez IM, Liao W. Ocular co-morbidities of atopic dermatitis. Part II: Ocular disease secondary to treatments. Am J Clin Dermatol. 2019. https://doi. org/10.1007/s40257-019-00465-3.

33. Zirwas MJ, Wulff K, Beckman K. Lifitegrast add-on treatment for dupilumab-induced ocular surface disease (DIOSD): a novel case report. JAAD Case Rep. 2019;5(1):34-6.

34. Rial MJ, Barroso B, Rodriguez-Bermejo C, Sastre J. Letter regarding "Conjunctivitis occurring in atopic dermatitis patients treated with dupilumab — clinical characteristics and treatment". J Allergy Clin Immunol Pract. 2019;7(2):753.

35. Aszodi N, Thurau S, Seegraber M, de Bruin-Weller M, Wollenberg A. Management of dupilumab-associated conjunctivitis in atopic dermatitis. J Dtsch Dermatol Ges. 2019;17(5):488-91. 